\title{
Fingerprint Image Enhancement Based on Second Directional Derivative of the Digital Image
}

\author{
Marius Tico \\ Nokia Research Center, P.O. Box 100, FIN-33721 Tampere, Finland \\ Email:marius.tico@nokia.com \\ Vesa Onnia \\ Institute of Digital and Computer Systems, Tampere University of Technology, P.O. Box 553, FIN-33101 Tampere, Finland \\ Email:vesa.onnia@tut.fi \\ Pauli Kuosmanen \\ Institute of Signal Processing, Tampere University of Technology, P.O. Box 553, FIN-33101 Tampere, Finland \\ Email:pauli.kuosmanen@tut.fi \\ Received 16 October 2001 and in revised form 10 April 2002

\begin{abstract}
This paper presents a novel approach of fingerprint image enhancement that relies on detecting the fingerprint ridges as image regions where the second directional derivative of the digital image is positive. A facet model is used in order to approximate the derivatives at each image pixel based on the intensity values of pixels located in a certain neighborhood. We note that the size of this neighborhood has a critical role in achieving accurate enhancement results. Using neighborhoods of various sizes, the proposed algorithm determines several candidate binary representations of the input fingerprint pattern. Subsequently, an output binary ridge-map image is created by selecting image zones, from the available binary image candidates, according to a MAP selection rule. Two public domain collections of fingerprint images are used in order to objectively assess the performance of the proposed fingerprint image enhancement approach.
\end{abstract}

Keywords and phrases: fingerprints, image enhancement, image derivative, facet model, ridge pattern.

\section{INTRODUCTION}

Fingerprints are graphical ridge patterns present on human fingers, which, due to their uniqueness and permanence, are among the most reliable human characteristics that can be used for people identification $[1,2]$. A common hypothesis, confirmed by the experience accumulated during a century of forensic use of fingerprints, is that certain local structures derived from the fingerprint ridges, called minutiae, are able to capture the invariant and discriminatory information present in the fingerprint image.

Several factors like the presence of scars, variations of the pressure between the finger and acquisition sensor, worn artifacts, the environmental conditions during the acquisition process, and so forth, can dramatically affect the quality of the acquired fingerprint image. Since minutiae depend on fine details of the ridge pattern, their extraction can become notoriously difficult if the noise generated by the factors described above is not substantially reduced. The main goals of a fingerprint image enhancement algorithm are (i) to reduce the noise present in the image, and (ii) to detect the fingerprint ridges. An input gray-scale fingerprint image is thereby transformed by the enhancement algorithm into a binary representation of the ridge pattern, called binary ridge-map image.

Inspecting a fingerprint image we may note that the image pixels located on fingerprint ridges usually exhibit lower gray level intensities than the image pixels located on the fingerprint valleys. An attempt to enhance the image would be to classify image pixels as ridge or valley pixels by comparing their intensities with a certain threshold intensity value. Unfortunately, such a simple point operation approach fails to reduce the noise present in the image because it does not take into consideration the strong correlation that exists between neighborhood intensity values in the fingerprint image pattern.

In general, it is quite difficult to model the noise present in the fingerprint image, but fortunately, the signal is fairly simple to characterize due to the flow of fingerprint ridges whose orientations are slowly changing in the fingerprint pattern. Taking for instance a small local area in the 


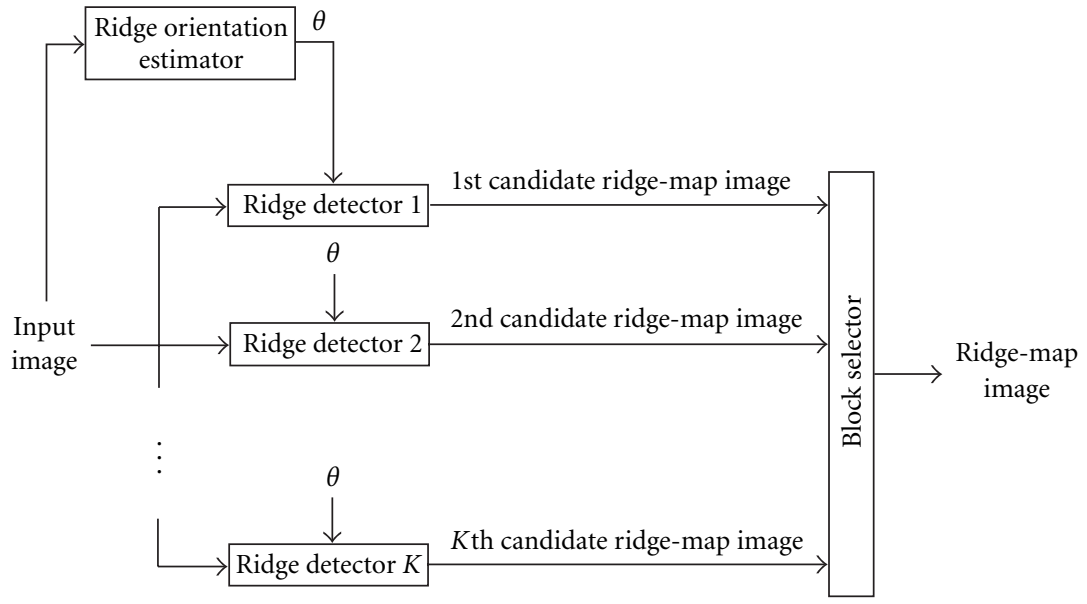

FIgURE 1: The diagram of the fingerprint image enhancement algorithm.

fingerprint image we may note that the ridge orientation as well as the ridge period are maintained almost constant inside this area, whereas the noise present in the image does not exhibit such regularities. The vast majority of the fingerprint enhancement methods proposed in the literature are based on this observation. The basic idea being to design adaptive filters attuned to the corresponding orientation and ridge period in each image region.

In the method proposed by Ratha et al. in [3] the image is smoothed using a one-dimensional averaging mask oriented along the local ridge orientation, and the fingerprint ridges are detected as local minima of the gray-level projection waveform along a scan line perpendicular to the local ridge orientation.

Jain et al. [4] proposed to accentuate local minima intensity values along a direction normal to the local ridge orientation by convolving the fingerprint image with two masks aligned to the ridge orientation. Subsequently, a binary ridge-map image is obtained by comparing the intensity values of the pixels in the two convolved images with a certain threshold value.

The enhancement algorithm proposed in [5] by Hong et al., relies on Gabor filters of size $11 \times 11$ pixels tuned to the ridge orientation and ridge period priorly estimated in nonoverlapping image blocks of $16 \times 16$ pixels.

Sherlock et al. [6] proposed a directional Fourier domain filtering for fingerprint enhancement. They design a set of 16 directional filters tuned on different orientations between 0 and $\pi$. Applying each filter onto the entire fingerprint image they obtain 16 filtered images. Next, the value of each pixel in the enhanced image is selected from one of the 16 filtered images based on the ridge orientation in the neighborhood of that pixel. Finally, the binary ridge-map image is obtained by a locally adaptive thresholding technique.

Instead of designing filters tuned on corresponding spatial frequency of each image region, Willis and Myers proposed in [7] to use as a filter directly the magnitude of the Fourier transform of the local image region. This magnitude already exhibits most of the qualities required from a properly designed enhancement filter since it has a dominant component at the corresponding ridge orientation and frequency, and on the other hand, due to the noise irregularity it exhibits small other components.

\subsection{Overview of the proposed approach}

The diagram of the fingerprint image enhancement algorithm proposed in this paper is shown in Figure 1. Using an estimate of the local ridge orientation we compute a set of $K$ candidate binary ridge-map images, each of them being tuned onto a restricted range of ridge periods. If the ridge period does not change significantly over the input fingerprint image, then one of the $K$ candidate binary ridge-map images is the one sought, and hence it will be selected as the output of the enhancement algorithm. On the other hand, if the ridge period exhibits significantly different values inside the same image, then no one of the $K$ candidates is able to accurately represent the entire ridge pattern. Nevertheless, since the candidate images are calculated for different ranges of the ridge period, an accurate binary-ridge-map image can be obtained by combining image zones selected from different candidates. To do this, we consider a division of the entire image in nonoverlapping image blocks, inside which one can assume an almost constant ridge period. Finally, the output binary ridge-map image is constructed by selecting the best binary representation of each image block among the $K$ corresponding blocks in the available set of candidate binary images.

In the following sections, we will describe in detail the proposed enhancement algorithm as well as the experimental results performed on two public domain collections of fingerprint images. Section 2 presents the approach used to calculate the candidate binary ridge-map images based on the sign of the second directional derivative of the digital image. The selection criterion used to identify the best binary representation of each image block is derived in Section 3. Experimental results and comparisons are presented in Section 4 of the paper, and some concluding remarks are presented in Section 5. 


\section{THE COMPUTATION OF A CANDIDATE BINARY RIDGE-MAP IMAGE}

Suppose that we construct a one-dimensional sequence by collecting the intensity values of the pixels located along a short line segment orthogonal to the local ridge orientation, as shown in Figure 2. The sequence usually exhibits an almost sinusoidal shape with low, and high intensity values corresponding to pixels located on the ridges, and on the valleys intersected by our segment, respectively. The discrimination between ridge and valley pixels can be thereby performed based on the sign of the second derivative of the onedimensional sequence. The positive, and negative values of this derivative correspond to pixels located on fingerprint ridges, and valleys, respectively.

Based on this observation, we detect the fingerprint ridges in those image pixels where the second directional derivative along the direction orthogonal to the local ridge orientation is positive.

Let $g(i, j)$ denote the value of the gray-level intensity at the pixel $(i, j)$ of the image, where $i$ denotes the horizontal coordinate that increases from left to right, and $j$ denotes the vertical coordinate that increases from bottom to up. Also, let $\theta \in[0, \pi)$ denote the local ridge orientation with respect to the horizontal axis, whose estimator is presented in Appendix A. The second derivative of the intensity surface along the direction $\mathbf{v}=[-\sin \theta \cos \theta]^{T}$, orthogonal to the local ridge orientation is given by

$$
\begin{aligned}
g_{\mathbf{v}}^{\prime \prime}(i, j)= & g^{(2,0)}(i, j) \sin ^{2} \theta+g^{(0,2)}(i, j) \cos ^{2} \theta \\
& -g^{(1,1)}(i, j) \sin 2 \theta,
\end{aligned}
$$

where $g^{(p, q)}(i, j)$ denotes the $(p+q)$ th partial derivative of the discrete intensity surface at site $(i, j), p$ along the horizontal axis and $q$ along the vertical axis.

The second partial derivatives of the discrete intensity surface can be approximated using a facet model [8]. A continuous surface $z=f(x, y)$ is fitted over the intensity values of image pixels located in a $(2 L+1) \times(2 L+1)$ window centered at $(i, j)$. Next, the partial derivatives of the discrete intensity surface at site $(i, j)$ are approximated by the corresponding partial derivatives of the continuous surface at $(0,0)$

$$
\left.g^{(p, q)}(i, j) \approx \frac{\partial^{p+q} f}{\partial x^{p} \partial y^{q}}\right|_{x=0, y=0} .
$$

Let us assume the following parametric form for the continuous surface:

$$
f(x, y)=\sum_{t_{1}=0}^{T} \sum_{t_{2}=0}^{T} c_{t_{1}, t_{2}} h_{t_{1}}(x) h_{t_{2}}(y),
$$

where $\left\{c_{t_{1}, t_{2}}: 0 \leq t_{1}, t_{2} \leq T\right\}$ denotes the set of unknown parameters, and $h_{t}$ for $0 \leq t \leq T$ are $T+1$ continuous onedimensional real functions defined on $[-L, L]$. As shown in [9], using a least squares estimator of the unknown parameters the partial derivative (2) can be approximated by

$$
g^{(p, q)}(i, j) \approx \sum_{c=-L}^{L} \mathbf{f}_{p, L}(c)\left(\sum_{r=-L}^{L} g(i-c, j-r) \mathbf{f}_{q, L}(r)\right),
$$

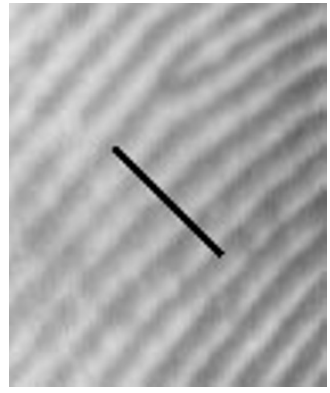

(a)

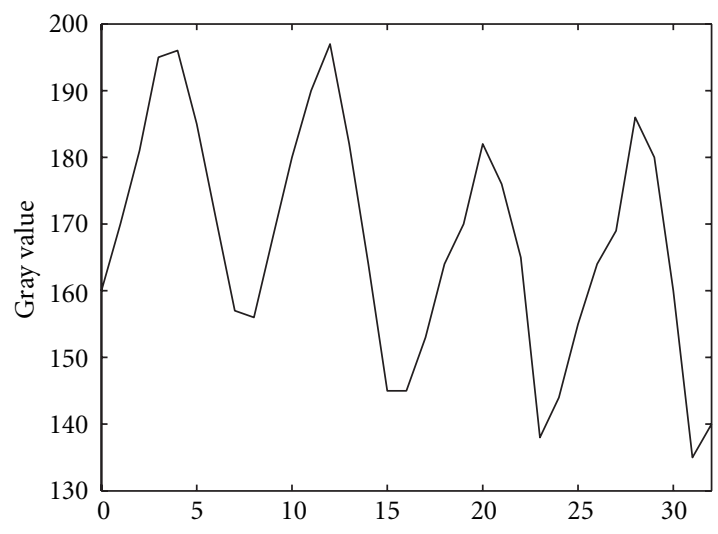

(b)

FIgURE 2: The intensity values of the image pixels located along a short line segment orthogonal to the local ridge orientation: (a) fingerprint image; (b) the waveform of intensity values of the selected pixels.

where $\mathbf{f}_{p, L}$ and $\mathbf{f}_{q, L}$ are the impulse responses of onedimensional finite impulse response filters of length $2 L+1$, that act along the image rows and columns. A useful property is that the values of the $2 L+1$ filter coefficients do not depend on image data. Consequently, they can be computed beforehand once the functions $\left\{h_{t}: 0 \leq t \leq T\right\}$ as well as the value of $L$, are decided. In this work, we model each function $h_{t}$ as a Chebyshev polynomial ${ }^{1}$ of order $t$. Using all polynomials up to the cubic ones (i.e., $T=3$ ), we can derive, in a closed form, the formulas for the filter coefficients that are shown as follows:

$$
\begin{aligned}
& \mathbf{f}_{0, L}(\ell)=\frac{3\left(3 L^{2}+3 L-1-5 \ell^{2}\right)}{(2 L+1)(2 L-1)(2 L+3)}, \\
& \mathbf{f}_{1, L}(\ell)=\frac{-5 \ell\left(15 L^{4}+30 L^{3}-15 L+5-21 L^{2} \ell^{2}-21 L \ell^{2}+7 \ell^{2}\right)}{L(L+1)(2 L+1)(L-1)(2 L-1)(L+2)(2 L+3)}, \\
& \mathbf{f}_{2, L}(\ell)=\frac{30\left(3 \ell^{2}-L^{2}-L\right)}{L(L+1)(2 L+1)(2 L-1)(2 L+3)} .
\end{aligned}
$$

\footnotetext{
${ }^{1}$ The reader may consult [8] by Haralick where a detailed discussion concerning the use of discrete Chebyshev polynomials for the approximation of partial derivatives of the digital images is presented.
} 


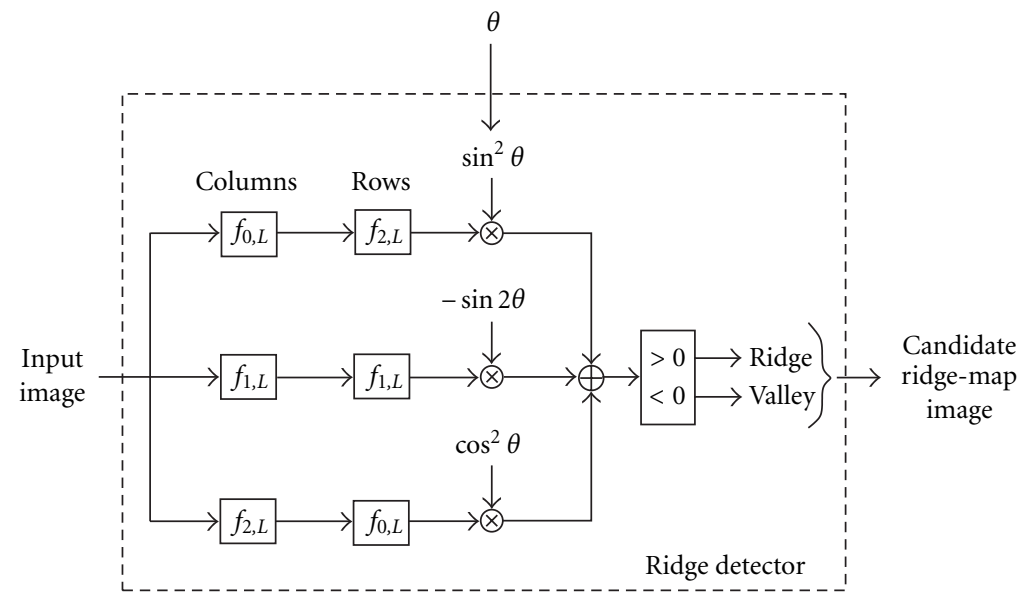

Figure 3: The computation of a candidate ridge-map image using precomputed one-dimensional filters of the same size (i.e., $2 L+1$ taps) over the entire image.

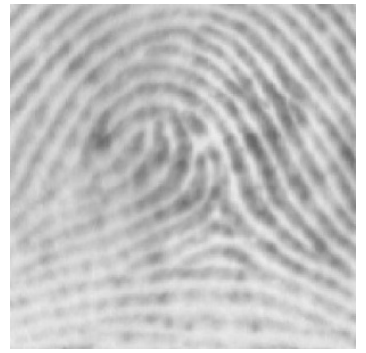

(a)

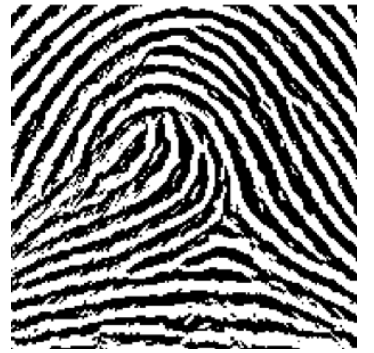

(b)

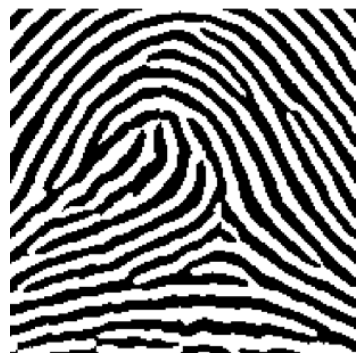

(c)

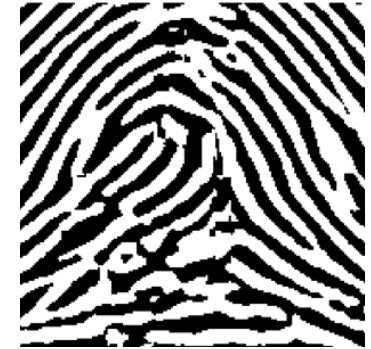

(d)

FIgURE 4: Binary images obtained for different sizes of the approximation window: (a) the original gray-scale image, (b) the binary representations obtained with $L=2$, (c) $L=4$, and (d) $L=10$.

The diagram of the ridge detection algorithm using a set of precomputed filters for a certain value of $L$ is shown in Figure 3. The directional derivative (1) is calculated as a weighted sum of the three filtered versions of the image, where the weights are generated based on the local ridge orientation. A candidate binary ridge-map image is obtained based on the sign of the directional derivative (1). The entire procedure has a small computational complexity since it is performed using only one-dimensional convolutions along the image rows and columns.

If the size $(2 L+1) \times(2 L+1)$ of the approximation window is selected in accordance with the ridge period, then the algorithm is able to reduce the noise and accurately detect the fingerprint ridges in the image, as exemplified in Figure 4c. Nevertheless, if $L$ is too small (Figure $4 \mathrm{~b}$ ), or too large (Figure $4 \mathrm{~d}$ ), then the algorithm fails to produce an accurate binary ridge-map image. On the one hand, for small values of $L$ there is only a small number of pixels in the approximation window, and hence the continuous surface $z=f(x, y)$ may overfit the few intensity values present in the window being unable to cancel the noise. On the other hand, for large values of $L$, the surface $z=f(x, y)$ may be too simple for fitting the ripples of the fingerprint ridges included in the approximation window, and hence it may alter the signal.

The size of the approximation window is thereby critical for the enhancement algorithm, and its value must be selected in accordance with the local ridge period. Our solution consists of computing $K$ candidate binary ridge-map images using the above algorithm with different values of $L$ (i.e., $L_{1}<L_{2}<\cdots<L_{K}$ ), following then to construct the output binary ridge-map image by selecting image regions from different candidates. To do this, we divide the input image as well as the $K$ candidate images in nonoverlapping blocks of size $W \times W$ pixels. Assuming that the ridge period does not change significantly inside an image block, we may expect that an accurate binary representation of the block will be found into one of the $K$ candidate binary ridge-map images.

\section{THE SELECTION OF THE BEST BINARY REPRESENTATION}

In this section, we introduce the selection criterion used to identify the best binary representation of each image block among the $K$ available candidate representations. Without 
any loss of generality, one may consider that the $N=W^{2}$ image pixels located inside an image block are indexed by a single scalar value $n \in\{1,2, \ldots, N\}$. The original gray-scale image block is regarded as a random field $\mathbf{G}=$ $\left\{G_{1}, G_{2}, \ldots, G_{N}\right\}$, where $G_{n}(1 \leq n \leq N)$ is the random variable that models the gray-level intensity of the $n$th pixel. Similarly, the binary representation of the image block is thought as a random field $\mathbf{B}=\left\{B_{1}, B_{2}, \ldots, B_{N}\right\}$. The observed image block is thereby a realization of $\mathbf{G}$ denoted here by $\mathbf{g}=\left\{g_{1}, g_{2}, \ldots, g_{N}\right\}$, whereas the $K$ candidate binary representations are realizations of $\mathbf{B}$ denoted by $\mathbf{b}_{k}=$ $\left\{b_{1}^{(k)}, b_{2}^{(k)}, \ldots, b_{N}^{(k)}\right\}$, where $1 \leq k \leq K$.

The problem that we are facing is the following: given an observed gray-scale image block $\mathbf{g}$ and the set of its $K$ binary representations $\left\{\mathbf{b}_{1}, \mathbf{b}_{2}, \ldots, \mathbf{b}_{K}\right\}$, select the best binary representation that describes the fingerprint ridges present in $\mathbf{g}$. In our work we consider that the best binary representation is the one that has the maximum a posteriori probability given the observation. Also, each one of the $K$ binary representations is thought as a model, based on which, the observed data could have been generated.

Let $P\left(\mathbf{b}_{k} \mid \mathbf{g}\right)$ denote the posterior probability of $\mathbf{b}_{k}$. From Bayes' theorem we have

$$
P\left(\mathbf{b}_{k} \mid \mathbf{g}\right)=\frac{p\left(\mathbf{g} \mid \mathbf{b}_{k}\right) P\left(\mathbf{b}_{k}\right)}{p(\mathbf{g})},
$$

where $p\left(\mathbf{g} \mid \mathbf{b}_{k}\right)$ is the probability density function of the observed data given that it is generated by the binary representation $\mathbf{b}_{k}, P\left(\mathbf{b}_{k}\right)$ is the prior probability of $\mathbf{b}_{k}$, and $p(\mathbf{g})$ is the probability density function of the data. Since

$$
p(\mathbf{g})=\sum_{k=1}^{K} p\left(\mathbf{g} \mid \mathbf{b}_{k}\right) P\left(\mathbf{b}_{k}\right)
$$

has the same value for all $k \in\{1,2, \ldots, K\}$, it does not influence the selection of a certain binary representation. Taking negative logarithm in (6), and dropping the factor $p(\mathbf{g})$ yields the following criterion to minimize

$$
C_{k}=-\log p\left(\mathbf{g} \mid \mathbf{b}_{k}\right)-\log P\left(\mathbf{b}_{k}\right) .
$$

It is worth noting that the criterion (8) can be interpreted as a code length since the first term can be thought as the ideal code length of the data given that it is generated by the model $\mathbf{b}_{k}$, and the second term can be thought as the ideal code length of the model itself. The first term, called from now on prediction term, expresses the ability of a certain model to predict the observed data, whereas the second term, called here prior term, expresses our prior preference toward a certain model. In the following, we derive the expressions of these two terms for our problem.

\subsection{The prediction term}

In order to compute the prediction term we assume that the gray-level intensity of the $n$th pixel is a random variable $G_{n}$ that has a conditionally Gaussian distribution given a realization of $B_{n}$

$$
G_{n} \sim \begin{cases}\mathcal{N}\left(\mu_{0}, v_{0}\right) & \text { if } b_{n}=0, \\ \mathcal{N}\left(\mu_{1}, v_{1}\right) & \text { if } b_{n}=1,\end{cases}
$$

where $\mathcal{N}(\mu, v)$ denotes the normal distribution of mean $\mu$, and variance $v$.

Assuming that the random variables $G_{n}(1 \leq n \leq N)$ are conditionally independent given the random field $\mathbf{B}$, we have

$$
p\left(\mathbf{g} \mid \psi_{k}, \mathbf{b}_{k}\right)=\prod_{n=1}^{N} p\left(g_{n} \mid \psi_{k}, b_{n}^{(k)}\right),
$$

where $\psi_{k}=\left[\begin{array}{lll}\mu_{0}^{(k)} & \mu_{1}^{(k)} v_{0}^{(k)} v_{1}^{(k)}\end{array}\right]^{T}$ denotes the parameter vector.

The marginal density of the data given the model, can be determined by assuming a prior probability density function $p\left(\psi_{k}\right)$ of the parameter vector

$$
p\left(\mathbf{g} \mid \mathbf{b}_{k}\right)=\int p\left(\mathbf{g} \mid \psi_{k}, \mathbf{b}_{k}\right) p\left(\psi_{k}\right) d \psi_{k} .
$$

Choosing an uninformative prior probability density function $p\left(\psi_{k}\right)$ such that not to bias our result, and assuming that the number of samples $N$ is large, we can use an asymptotic approximation for the integral (11) as those presented in [10], or in [11]. The logarithm of the density (11) can be thereby expressed as

$$
-\log p\left(\mathbf{g} \mid \mathbf{b}_{k}\right) \approx-\log p\left(\mathbf{g} \mid \hat{\psi}_{k}, \mathbf{b}_{k}\right)+\frac{1}{2} \log \left|\mathbf{I}\left(\hat{\psi}_{k}\right)\right|,
$$

where $\hat{\psi}_{k}$ is the maximum likelihood estimate of the parameter vector $\left(\hat{\psi}_{k}=\arg \max _{\psi} p\left(\mathbf{g} \mid \psi, \mathbf{b}_{k}\right)\right)$, and $\left|\mathbf{I}\left(\hat{\psi}_{k}\right)\right|$ is the determinant of the Fisher information matrix evaluated at $\hat{\psi}_{k}$ (see [12]).

Evaluating (12) for the distribution (10), and dropping all terms that do not depend of $k$ (see Appendix B), we get the following approximation for the prediction term:

$$
\begin{aligned}
-\log p\left(\mathbf{g} \mid \mathbf{b}_{k}\right) \approx & \log \left(N_{0}^{(k)} N_{1}^{(k)}\right)+\frac{N_{0}^{(k)}-3}{2} \log \hat{v}_{0}^{(k)} \\
& +\frac{N_{1}^{(k)}-3}{2} \log \hat{v}_{1}^{(k)},
\end{aligned}
$$

where $N_{0}^{(k)}$ and $N_{1}^{(k)}$ denote the number of zero and one pixels, respectively that are contained into the binary representation $\mathbf{b}_{k} ; \hat{v}_{0}^{(k)}$ and $\hat{v}_{1}^{(k)}$ denote the estimated variances of the observed intensity values $g_{n}$ corresponding to binary values $b_{n}^{(k)}=0$, and $b_{n}^{(k)}=1$, respectively.

\subsection{The prior term}

As we mentioned in the introduction, the noise present in the fingerprint image is quite difficult to model but, on the other hand, the data is relatively simple to characterize due to the redundancy of parallel ridges. The prior term models our idea about a noise free binary representation of the observed ridge pattern. This term is meant to bias our preference 
toward those binary representations that exhibit specific characteristics which are likely to appear in a fingerprint pattern. For instance, in order to reduce the effect of various noisy factors (e.g., scars, cuts, smudgy regions) that may cause false ridge breaks as well as irregularities of the ridge profile, one may require that the binary representation of a certain image block to be smooth and unfragmented along the direction of the local ridge orientation.

In this work, we model the binary representation $\mathbf{B}$ of a certain image block as a two-dimensional Markov random field (MRF). For simplicity, as well as in order to reduce the computational load, we assume only horizontal and vertical spatial dependencies between adjacent pixels.

A pair of horizontally adjacent pixels is called horizontal clique, and similarly a pair of vertically adjacent pixels is called vertical clique. ${ }^{2}$ In order to preserve the spatial continuity in the binary image along the local ridge orientation $(\theta)$, we define the potential of a clique as follows:

$$
V\left(b_{i}, b_{j}\right)= \begin{cases}\cos ^{2} \theta & \text { if }(i, j) \in \mathscr{H} \text { and } b_{i} \neq b_{j}, \\ \sin ^{2} \theta & \text { if }(i, j) \in \mathscr{V} \text { and } b_{i} \neq b_{j} \\ 0 & \text { if } b_{i}=b_{j}\end{cases}
$$

where $\mathscr{H}$ and $\mathscr{V}$ denote the collections of horizontal and vertical cliques, respectively.

The probability mass function of $\mathbf{B}$ can be assumed to have the form

$$
P(\mathbf{B}=\mathbf{b})=\frac{1}{Z} \exp \left(-\rho \sum_{(i, j) \in \mathscr{C}} V\left(b_{i}, b_{j}\right)\right),
$$

where $\mathscr{C}=\mathscr{H} \cup \mathscr{V}$, and $Z$ is a normalizing constant that guarantees $\sum_{k=1}^{K} P\left(\mathbf{b}_{k}\right)=1$, and hence it does not influence the selection of the best binary representation. Taking logarithm in (15) and dropping $Z$ we obtain an approximation of the prior term

$$
-\log P\left(\mathbf{b}_{k}\right) \approx \rho \sum_{(i, j) \in \mathscr{C}} V\left(b_{i}^{(k)}, b_{j}^{(k)}\right) .
$$

Finally, using formulas (13) and (16), the selection criterion (8) can be expressed as

$$
\begin{aligned}
C_{k}= & \log \left(N_{0}^{(k)} N_{1}^{(k)}\right)+\frac{N_{0}^{(k)}-3}{2} \log \hat{v}_{0}^{(k)} \\
& +\frac{N_{1}^{(k)}-3}{2} \log \hat{v}_{1}^{(k)}+\rho \sum_{(i, j) \in \mathscr{C}} V\left(b_{i}^{(k)}, b_{j}^{(k)}\right),
\end{aligned}
$$

where the parameter $\rho$ is taken such that to balance the relative importance of the two terms (i.e., prediction term and prior term) in the selection criterion. In our experiments we have used $\rho=2$ which seems to work well.

\footnotetext{
${ }^{2}$ The reader may review the basic definitions related with the concept of clique in the paper [13] by Derin and Elliott.
}

\section{RESULTS}

The fingerprint image enhancement algorithm presented in this paper has been tested on two public domain collections of fingerprint images. The first collection (DB1) comprises 400 live-scan images of 50 fingers (8 impressions per finger) selected from the first database described in [14]. The second experimental data collection (DB2) comprises 168 images of 21 fingers (8 impressions per finger) and it has been created by Biometric Systems Lab., University of Bologna, Cesena, Italy (www2.csr.unibo.it/research/biolab).

The values of the algorithmic parameters used in our implementation have been empirically selected at $W=16$, $\rho=2$, and a number of $K=4$ candidate binary ridge-map images, computed for $L \in\{2,3,4,5\}$, have been used.

A subjective evaluation of the enhancement algorithm has been performed by visually inspecting several enhancement results. Two examples of fingerprint image enhancement are shown in Figure 5. From these examples we may note that the enhancement algorithm is able to accurately detect the fingerprint ridges present in the input image.

The ability of the proposed selection criterion (17) to identify the best binary representation among the available candidates is illustrated by the examples shown in Figure 6 . In this examples, the $K=2$ candidate images (Figures 6b and $6 c$ ) have been calculated using filters of length 7 and 11 taps, respectively. We note that neither one of the candidate images is able to accurately represent the entire fingerprint pattern present at the input. Nevertheless, both candidates exhibit good representations of certain image zones, which are selected based on the proposed criterion, and eventually included into the output binary ridge-map images shown in Figure 6d.

The main objective of an enhancement algorithm is to process an image such that the result is more suitable than the original image for a specific application [15]. The fingerprint enhancement algorithm is meant to process the input image such that to facilitate a more accurate minutiae detection, and hence to improve, in the end, the performance of the fingerprint verification system. If the same algorithms of minutiae detection, and fingerprint matching are used in combination with different fingerprint image enhancement algorithms, then the estimated matching performance constitutes an objective comparison criterion between the given enhancement methods. In our experiments we used the approach of minutiae detection, described in [16], in conjunction with the minutiae-based fingerprint matching procedure introduced in [17].

The matching performance, expressed in terms of equal error rate (EER), achieved on both data collections using only one candidate image, and multiple candidate images are shown in Table 1.

The fingerprint images in DB1 have been acquired at a larger resolution than the images included in DB2, and hence they also exhibit a larger average ridge period. This is well reflected by the matching performance achieved when the enhancement algorithm relies only on a single candidate binary ridge-map image that is computed using filters of length 


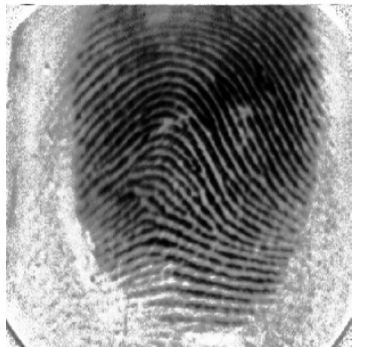

(a)

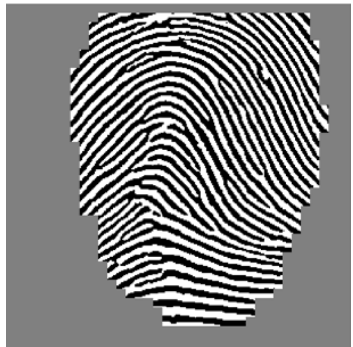

(b)

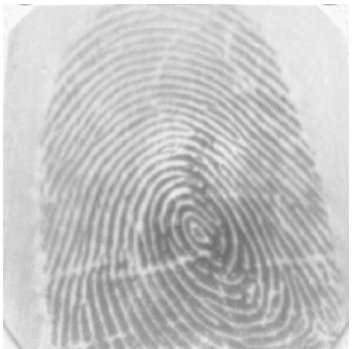

(c)

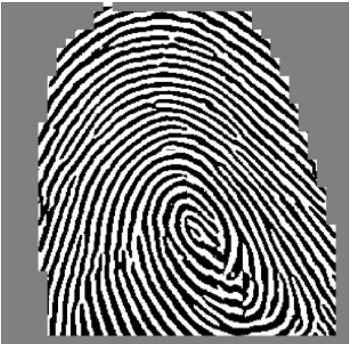

(d)

FIGURE 5: Example of fingerprint image enhancement: the input images (a) and (c); the enhanced images (b) and (d).
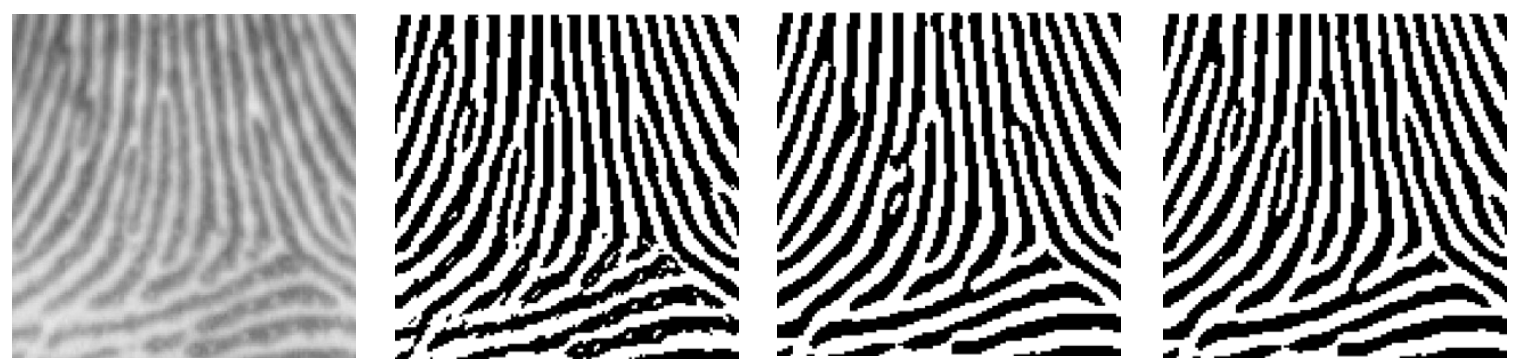

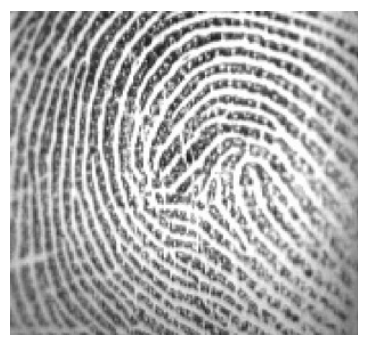

(a)

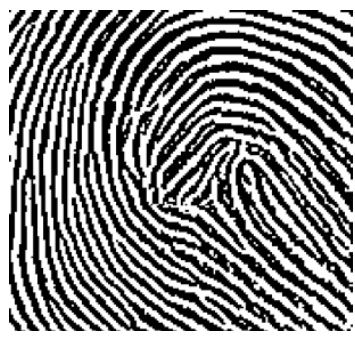

(b)

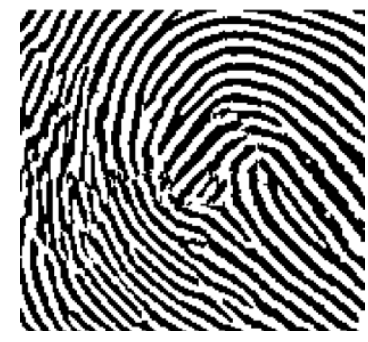

(c)

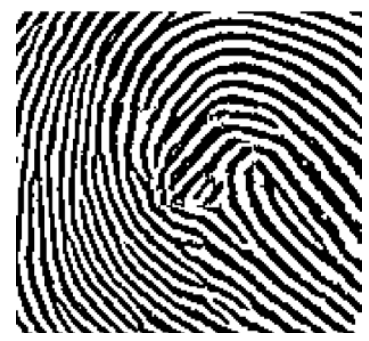

(d)

FIGURE 6: Examples of binary ridge-map computation using two image candidates: (a) the input fingerprint images, (b) the first candidate images, (c) the second candidate images, and (d) the output binary ridge-map images.

$2 L+1$ taps over the entire image. For instance, we note that a good choice for DB1 is to calculate the output binary ridgemap image using only a single candidate image computed for $L=5$, whereas the same choice would give poor performance on DB2. The results reveal the ability of the proposed algorithm to adapt at different values of the ridge period in the two image collections, since it achieves the best performance on both collections, without changing the values of the algorithmic parameters specified above.

For comparison we implemented two fingerprint image enhancement algorithms proposed in the literature. One algorithm (A) was adopted from [7], and the other algorithm (B) was implemented after [3].

The receiver operating characteristic (ROC) curves estimated on DB1, when each one of the three fingerprint image enhancement algorithms is embedded into the verification system, are shown in Figure 7. We note that the results are in favor of our enhancement algorithm, revealing an improvement in matching performance when this algorithm is included into the fingerprint verification system.

A visual comparison between the three algorithms is illustrated in Figure 8. The fingerprint images shown in this example belong to DB1 and exhibit different degree of image quality. Inspecting the enhancement results we note that the proposed approach is able to adapt quite well to the local geometry of the fingerprint ridges, and on the other hand it exhibits a smaller sensitivity to noisy artifacts (e.g., scars, contact pressure variations, worn artifacts) then the other two methods.

In practice, the execution time of a fingerprint image enhancement routine must be within a few seconds. We evaluated the execution time of our algorithm on a Sun ULTRA 1 Workstation using for experiment the fingerprint images from DB1. The average execution times of different operations performed by our enhancement algorithm are shown in Table 2. Using the same experimental framework, the execution times evaluated for the methods A and B were 2.8 and 3.2 seconds, respectively. 


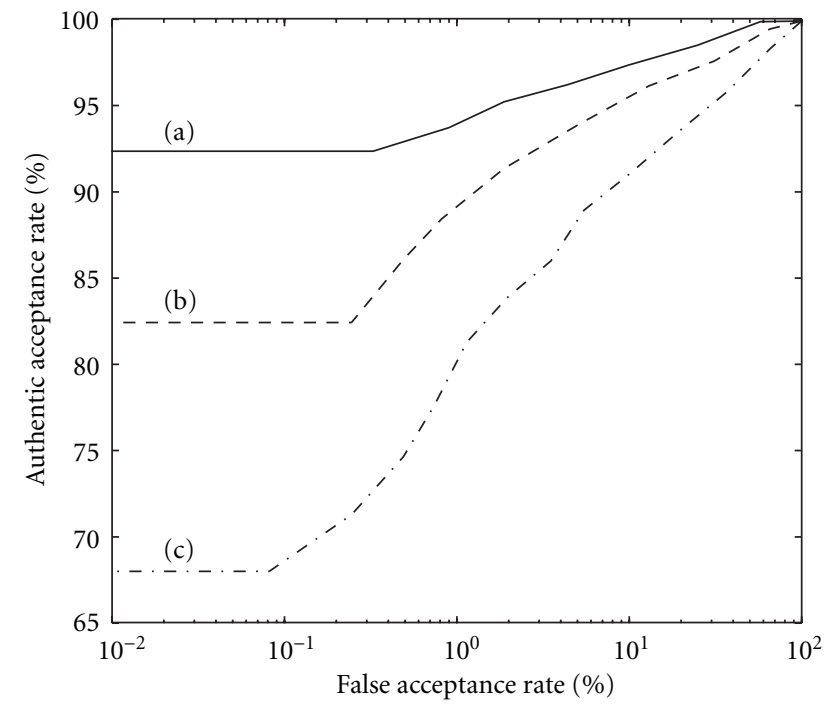

FIGURE 7: ROC-curves for different enhancement algorithms embedded into the verification system: (a) our algorithm, (b) the algorithm A, and (c) the algorithm B.

\section{CONCLUSIONS}

In this paper, we introduced a novel approach to fingerprint image enhancement. Our enhancement algorithm calculates a binary representation of the fingerprint pattern based on the sign of second directional derivative of the digital image. A cubic facet model was used in order to approximate the derivatives of the digital image at each pixel based on the discrete intensity values of image pixels located in a certain approximation window. It has been shown by examples that the size of the approximation window should be selected in accordance to the local ridge period in order to obtain an accurate binary representation of the fingerprint pattern. We proposed an adaptive scheme that creates the output binary ridge-map image by selecting image blocks from a set of available binary image candidates that are computed using approximation windows of different sizes. For each image block the binary representation that has the maximum a posteriori probability given the observed gray-level fingerprint pattern is selected among the available candidates. The proposed method has been evaluated by visually inspecting several enhancement results. In addition, an objective evaluation was carried out by recording the improvement in matching performance achieved by a fingerprint verification system that includes the enhancement algorithm.

\section{APPENDICES}

\section{A. THE ESTIMATION OF THE LOCAL RIDGE ORIENTATION}

In order to implement the proposed enhancement algorithm we must establish an approach for estimating the dominant ridge orientation in different image regions. In our implementation we used the orientation estimator proposed by
TABLE 1: The matching performance estimated on DB1 and DB2 using one and multiple candidates binary ridge-map images.

\begin{tabular}{ccc}
\hline \multirow{2}{*}{$L$} & \multicolumn{2}{c}{ EER $(\%)$} \\
\cline { 2 - 3 } & DB1 & DB2 \\
\hline 2 & K candidate image \\
3 & 7.0 & 2.4 \\
4 & 5.0 & 1.0 \\
5 & 4.7 & 2.5 \\
\hline \multicolumn{2}{c}{$K=4$ candidate images } \\
\hline $2,3,4,5$ & 3.9 & 8.8 \\
\hline
\end{tabular}

TABLE 2: The average execution times of different operations performed by the enhancement algorithm on a Sun ULTRA 1 Workstation.

\begin{tabular}{cc}
\hline Operation & $\begin{array}{c}\text { Execution time } \\
\text { (seconds) }\end{array}$ \\
\hline The computation of candidate binary images & 2.1 \\
The selection of the best binary representation & 0.2 \\
\hline Total & 2.3 \\
\hline
\end{tabular}

Rao in [18], and we assumed that the ridge orientation is almost constant inside each image block of size $W \times W$ pixels. Denoting by $g_{x}(n)$ and $g_{y}(n)$ the horizontal and vertical projections of the intensity gradient vector in the $n$th pixel of an image block $\left(1 \leq n \leq N\right.$, where $\left.N=W^{2}\right)$, the dominant ridge orientation $(\theta \in[0, \pi))$ inside the given block is estimated with the formula

$$
\theta=\frac{1}{2} \arctan \left(\frac{2 \sum_{n=1}^{N} g_{x}(n) g_{y}(n)}{\sum_{n=1}^{N}\left(g_{x}^{2}(n)-g_{y}^{2}(n)\right)}\right)+\frac{\pi}{2},
$$

where arctangent is computed using two arguments and lies in the range $[-\pi, \pi)$.

\section{B. THE DERIVATION OF EQUATION (13)}

In order to derive the expression of the prediction term (13) we need to determine the two terms of (12) for the density (10). The log-likelihood function of the density (10) is given by

$$
\begin{aligned}
& \log p\left(\mathbf{g} \mid \psi_{k}, \mathbf{b}_{k}\right) \\
& \quad=-\frac{N}{2} \log (2 \pi)-\sum_{i=0}^{1}\left[\frac{N_{i}^{(k)}}{2} \log v_{i}^{(k)}-\frac{1}{2 v_{i}^{(k)}} \sum_{n \in S_{i}}\left(g_{n}-\mu_{i}^{(k)}\right)^{2}\right],
\end{aligned}
$$

where $S_{i}=\left\{n ; b_{n}^{(k)}=i\right\}$, for $i \in\{0,1\}$. Replacing the parameters with their maximum likelihood estimates we obtain the 

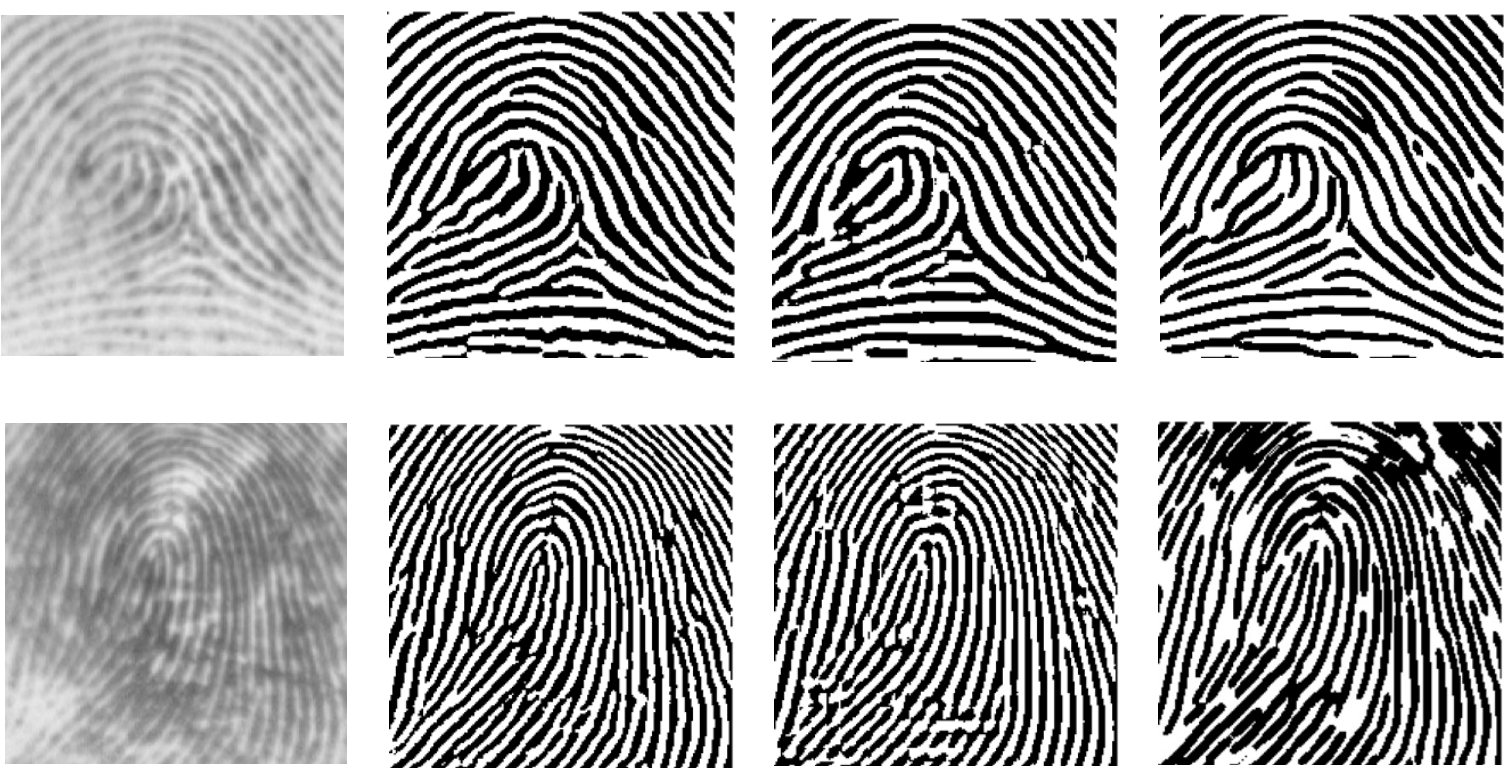

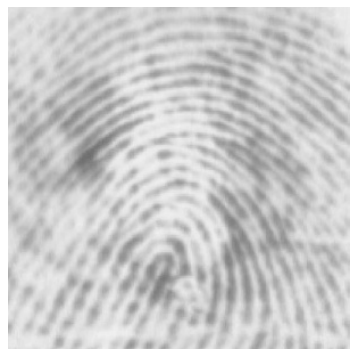

(a)

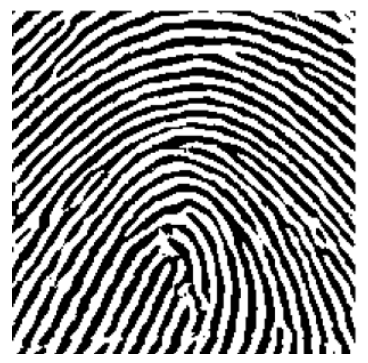

(b)

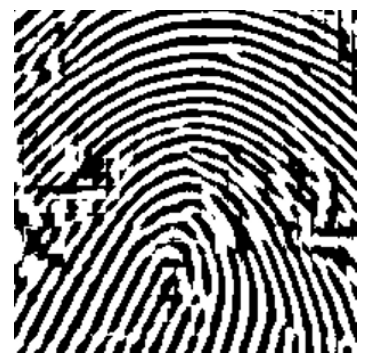

(c)

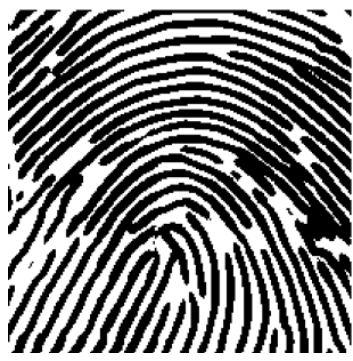

(d)

Figure 8: Examples of fingerprint image enhancement. The figure shows the input fingerprint patterns (column a), and the enhancement results achieved by our approach (column b), method A (column c), and method B (column d).

expression of the first term in (12)

$$
-\log p\left(\mathbf{g} \mid \hat{\psi}_{k}, \mathbf{b}_{k}\right)=\sum_{i=0}^{1} \frac{N_{i}^{(k)}}{2} \log \hat{v}_{i}^{(k)}+\frac{N}{2}[\log (2 \pi)+1] .
$$

Next, from (B.1) we can determine the Fisher information matrix that, in this case, is the $4 \times 4$ diagonal matrix

$$
\mathbf{I}\left(\psi_{k}\right)=\operatorname{diag}\left(\frac{N_{0}^{(k)}}{v_{0}^{(k)}}, \frac{N_{1}^{(k)}}{v_{1}^{(k)}}, \frac{N_{0}^{(k)}}{2\left(v_{0}^{(k)}\right)^{2}}, \frac{N_{1}^{(k)}}{2\left(v_{1}^{(k)}\right)^{2}}\right),
$$

whose determinant evaluated at $\hat{\psi}_{k}$, is included in the second term of (12)

$$
\frac{1}{2} \log \left|\mathbf{I}\left(\hat{\psi}_{k}\right)\right|=\sum_{i=0}^{1}\left[\log N_{i}^{(k)}-\frac{3}{2} \log \hat{v}_{i}^{(k)}\right]-\log 2 .
$$

Finally, replacing (B.2) and (B.4) in (12), and dropping all those terms which do not depend on $k$, we obtain the expression (13) of the prediction term.

\section{REFERENCES}

[1] H. C. Lee and R. E. Gaensslen, Advances in Fingerprint Technology, CRC Press, New York, USA, 1991.

[2] L. O'Gorman, "Fingerprint verification," in BiometricsPersonal Identification in Networked Society, A. K. Jain, R. Bolle, and S. Pankanti, Eds., pp. 43-64, Kluwer Academic, The Netherlands, November 1999.

[3] N. K. Ratha, S. Chen, and A. K. Jain, "Adaptive flow orientation-based feature extraction in fingerprint images," Pattern Recognition, vol. 28, no. 11, pp. 1657-1672, 1995.

[4] A. K. Jain, L. Hong, S. Pankanti, and R. Bolle, "An identityauthentication system using fingerprints," Proceedings of the IEEE, vol. 85, no. 9, pp. 1365-1388, 1997.

[5] L. Hong, Y. Wan, and A. K. Jain, "Fingerprint image enhancement: Algorithm and performance evaluation," IEEE Trans. on Pattern Analysis and Machine Intelligence, vol. 20, no. 8, pp. 777-789, 1998.

[6] B. G. Sherlock, D. M. Monro, and K. Millard, "Fingerprint enhancement by directional Fourier filtering," IEE ProceedingsVision, Image and Signal Processing, vol. 141, no. 2, pp. 87-94, 1994.

[7] A. J. Willis and L. Myers, "A cost-effective fingerprint recognition system for use with low-quality prints and damaged fingertips," Pattern Recognition, vol. 34, no. 2, pp. 255-270, 2001 
[8] R. M. Haralick, "Digital step edges from zero crossing of second directional derivatives," IEEE Trans. on Pattern Analysis and Machine Intelligence, vol. 6, no. 1, pp. 58-68, 1984.

[9] M. Tico and P. Kuosmanen, "Weighted least squares method for the approximation of directional derivatives," in Proc. IEEE Int. Conf. Acoustics, Speech, Signal Processing, vol. 3, pp. 1681-1684, Salt Lake City, Utah, USA, 2001.

[10] P. M. Djurić, "Asymptotic MAP criteria for model selection," IEEE Trans. Signal Processing, vol. 46, no. 10, pp. 2726-2735, 1998.

[11] S. M. Kay, Fundamentals of Statistical Signal ProcessingDetection Theory, vol. 2, Prentice-Hall, Englewood Cliffs, NJ, USA, 1998

[12] S. M. Kay, Fundamentals of Statistical Signal ProcessingEstimation Theory, vol. 1, Prentice-Hall, Englewood Cliffs, NJ, USA, 1993.

[13] H. Derin and H. Elliott, "Modeling and segmentation of noisy and textured images using Gibbs random fields," IEEE Trans. on Pattern Analysis and Machine Intelligence, vol. 9, no. 1, pp. 39-55, 1987.

[14] D. Maio, D. Maltoni, R. Cappelli, J. L. Wayman, and A. K. Jain, "FVC2000: Fingerprint verification competition," in Proc. 15th International Conference on Pattern Recognition, Barcelona, Spain, September 2000.

[15] R. C. Gonzalez and R. E. Woods, Digital Image Processing, Addison-Wesley, Reading, Mass, USA, 1992.

[16] M. Tico and P. Kuosmanen, "An algorithm for fingerprint image postprocessing," in Proc. 34th Asilomar Conference on Signals, Systems, and Computers, vol. 2, pp. 1735-1739, Pacific Grove, Calif, USA, 2000.

[17] M. Tico, On design and implementation of fingerprint-based biometric systems, Ph.D. thesis, Tampere University of Technology, Tampere, Finland, 2001.

[18] A. R. Rao, A Taxonomy for Texture Description and Identification, Springer-Verlag, New York, USA, 1990.

Marius Tico received his M.S. degree in computer science and the Ph.D. degree in electronics and telecommunications, both form Technical University of Cluj-Napoca, Romania, in 1993 and 1999, respectively. In 2001 he received the Doctor of Technology degree in signal and image processing from Tampere University of Technology, Finland. Between 1994 and 1997 he held a Teach-

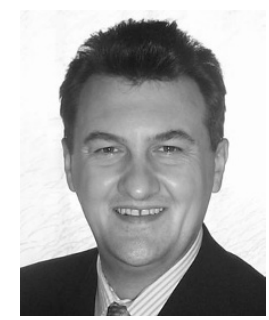
ing Assistant position at the Department of Electronics and Telecommunications of Technical University of Cluj-Napoca, Romania. From 1998 to 2001, he was a Research Engineer with the Institute of Signal Processing, Tampere University of Technology, Finland. Since 2001, he has been a Research Engineer at Nokia Research Center, Tampere, Finland. His research interests include signal and image processing, pattern recognition and biometrics.

Vesa Onnia received his M.S. and Licentiate degree in digital and computer systems science from Tampere University of Technology, Finland, in 1998 and 2001, respectively. $\mathrm{He}$ is currently a post graduate student in the Institute of Digital and Computer Systems at Tampere University of Technology. His research interests include image processing, biometrics, neural networks, and pattern recognition.
Pauli Kuosmanen received his B.S., M.S., and Licentiate degrees in mathematics from the University of Tampere, Finland, in 1989, 1991, and 1993, respectively. He received the Doctor of Technology degree in signal processing from the Tampere University of Technology in 1994. Since 1990 he has held various research and teaching positions at the University of Tampere and the Tampere University of Technology. Currently, he is a

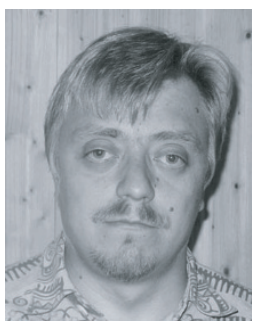
part-time Professor of signal processing. From August 2001 he has been working as a Director of New Technologies at the Research Center of Elisa Communications Corp. Prof. Kuosmanen has authored over 90 international journal and conference articles, two international book chapters, and he is a coauthor in the book Fundamentals of Nonlinear Digital Filtering. He has supervised several M.S. and Dr.Tech. students and has been leading tens of research projects related to signal and image processing, and digital media technology. 